\title{
Meconium aspiration syndrome: challenges and solutions
}

This article was published in the following Dove Press journal:

Research and Reports in Neonatology

16 August 2017

Number of times this article has been viewed

\section{Ankita Goel \\ Sushma Nangia}

Department of Neonatology, Lady Hardinge Medical College and

Kalawati Saran Children Hospital, Shaheed Bhagat Singh Marg,

New Delhi, India
Correspondence: Ankita Goel Kalawati Saran Children Hospital, Room No. I 18, Old Building, Shaheed Bhagat Singh Marg, New Delhi, India Tel +9| 97 I I 278490

Email ankitagoel.85@gmail.com
Abstract: Meconium aspiration syndrome (MAS) is an important cause of morbidity and mortality among term newborns. A result of antepartum or postpartum aspiration of meconium stained amniotic fluid (MSAF), MAS causes respiratory distress of varying severity, often complicated by air leaks or persistent pulmonary hypertension (PPHN). There has been a tremendous change in the concepts of pathophysiology and management of MAS over the last few decades. Routine endotracheal suctioning is no longer recommended in both vigorous and nonvigorous neonates with MSAF. Supportive management, along with newer therapies such as surfactant, inhaled nitric oxide, and high-frequency ventilation, has resulted in marked improvement in the overall outcome of MAS. The present review highlights the challenges in understanding the complex pathophysiology and optimal management approach to MAS. Potential future therapies and drugs in trial are also discussed briefly.

Keywords: meconium aspiration syndrome, endotracheal suction, surfactant, inhaled nitric oxide, high-frequency ventilation, persistent pulmonary hypertension

\section{Introduction}

Meconium aspiration syndrome (MAS) is a result of ante- or postpartum aspiration of meconium-stained amniotic fluid in term or near-term infants resulting in respiratory morbidity of varying severity. ${ }^{1}$ Despite rapid advances in diagnostic and therapeutic modalities over the past decades, meconium and its effects on fetus and neonates have remained a cause of significant worry for both the obstetrician and neonatologists. There are still widespread debates with varied opinions regarding the optimum obstetrical approach, resuscitative measures at birth and management of critically ill neonates with MAS. With the recognition of role of chronic in utero hypoxia and associated perinatal asphyxia in pathogenesis of MAS, routine endotracheal suctioning is no longer recommended even in nonvigorous infants with MAS. Advent of newer therapies like inhaled NO, surfactant, high-frequency ventilation and extracorporeal membrane oxygenation (ECMO) has resulted in tremendous improvements in MAS survival. This review highlights the current understanding of MAS pathophysiology and challenges faced in the optimal management of such patients.

\section{Definition}

MAS is defined as respiratory distress in a neonate born through meconium-stained amniotic fluid (MSAF) with characteristic radiological findings (hyperinflation and patchy opacities) whose symptoms cannot be explained otherwise. ${ }^{2}$ It is one of 
the most common causes of respiratory morbidity in term newborns requiring neonatal intensive care unit (NICU) stay. Traditionally, severity of MAS was graded on the basis of requirement of respiratory support as: 1) mild MAS, requiring $<40 \%$ oxygen for $<48$ hours, 2 ) moderate MAS, that requires more than $40 \%$ oxygen for more than 48 hours with no air leaks, and 3) severe MAS that requires assisted ventilation for more than 48 hours and is often associated with persistent pulmonary hypertension (PPHN). ${ }^{3}$ This classification, though widely followed, has a drawback of missing out on the categorization of neonates with MAS requiring ventilation for $<48$ hours. Hence, any requirement of mechanical ventilation and/or continuous positive airway pressure (CPAP) has been used as criteria for severe MAS in a few epidemiological studies (classifying them as MAS intubated and MAS nonintubated). ${ }^{4}$

\section{Epidemiology}

Fetal passage of meconium leading to MSAF complicates about $7 \%-20 \%$ of all pregnancies worldwide, the incidence being higher in blacks and south Asian ethnicity., ${ }^{3,5}$ The incidence also increases as the gestational age advances with reported frequencies at 37,40 , and $>42$ weeks being $3 \%$, $13 \%$, and $18 \%$ respectively. ${ }^{6}$ Apart from fetal maturation, various fetomaternal stress factors like maternal hypertension, oligohydramnios, maternal drug abuse (especially tobacco and cocaine), primigravidity, anemia, chorioamnionitis, prolonged labor, fetal distress, cord problems, and fetal growth retardation promote passage of meconium. ${ }^{7}$

Aspiration of meconium into fetal airways can occur in utero or during delivery resulting in MAS in $2 \%-9 \%$ cases of $\mathrm{MSAF}^{8}$ Of these, nearly half require mechanical ventilation, 15\%-20\% develop air leaks, and 5\%-12\% die. ${ }^{9}$ An estimated 25,000 to 30,000 cases and 1000 deaths related to MAS occur annually in the USA. ${ }^{10} \mathrm{~A}$ review of ten reports published from 1990 to 1998 showed a combined incidence rate of $13.1 \%$ for MSAF, $4.2 \%$ of MAS among MSAF, and $49.7 \%$ of MAS requiring ventilatory support with $4.6 \%$ mortality. ${ }^{11}$ A retrospective population-based survey from France between 2000 and 2007 demonstrated a global prevalence of MAS among MSAF as $2.29 \%$ with $36.9 \%$ of MAS infants having severe MAS (requiring mechanical ventilation/nasal CPAP). ${ }^{8}$ Varied studies have reported ethnicity (Pacific islander, Africans, and Asians), advanced gestational age, nonreassuring fetal heart rate, thick meconium, delivery by cesarean section, need for endotracheal intubation, the presence of meconium below the cords, and APGAR score $\leq 3$ at 1 minute as significant risk factors associated with the development of MAS in infants with MSAF. ${ }^{4,8,12}$

Of late, there are encouraging trends of a progressive decline in the incidence of MAS. In the USA, Yoder et al documented a fourfold decline in the incidence of MAS from $5.8 \%$ to $1.5 \%$ during $1990-1997$, attributable to $33 \%$ reduction in the incidence of births at $>41$ weeks of gestation. ${ }^{13}$ Similar decrease in incidence has been reported from multicenter trial in Australia and New Zealand (0.43-0.37 per 1000 live births between 1995 and 2002). ${ }^{4}$ This reduction in MAS incidence has been attributed to better obstetric practices, in particular, avoidance of post maturity, and expeditious delivery where fetal distress has been noted. In contrast, developing countries like India and Africa, with limited resources for fetal monitoring and delivery room care, still share a major burden of morbidity due to MAS.

\section{Pathophysiology}

Meconium is a sterile, odorless, blackish green material comprising intestinal and pancreatic secretions, desquamated cells, vernix caseosa, lanugo hair, bile pigments, amniotic fluid, and blood. It first appears in fetal intestine by approximately the 10th week of gestation and moves to colon by the 16th week. ${ }^{2}$ Under normal circumstances, passage of meconium from the fetus into amnion is prevented by the lack of intestinal peristalsis due to low motilin levels, tonic contraction of anal sphincter, and a terminal cap of viscous meconium. ${ }^{10}$ Transient parasympathetic neural stimulation because of head or cord compression, higher motilin levels, and increasing cholinergic innervation with advancing gestational age accounts for higher incidence of MSAF in term and post terms. ${ }^{14}$ Apart from fetal maturation, in utero stress with resultant fetal hypoxia and acidosis produces relaxation of anal sphincter, leading to meconium passage. ${ }^{10}$ In support of a chronic stress mechanism, Manning et al reported MSAF to occur twice more often if the biophysical profile score was $\leq 6$, as compared to $\geq 8 .{ }^{15}$ Erythropoietin levels were found to be increased in babies born through MSAF, possibly indicating chronic hypoxia contributing to meconium passage. ${ }^{16}$ However, few studies have documented the incidence of MSAF without preceding fetal distress or acidosis, indicating more robust research to elucidate the true mechanism of this association. ${ }^{17}$

Meconium in amniotic fluid can be aspirated during fetal gasping or in the initial breaths after delivery. Chronic in utero insult has been suggested to be responsible for severe cases of MAS, as opposed to acute peripartum events. ${ }^{18}$ Some studies concerning MAS have revealed that most 
cases of severe MAS are not causally related to the aspiration of meconium but rather are caused by other pathologic processes occurring in utero, primarily chronic asphyxia and infection. ${ }^{19}$

Despite substantial research, the pathophysiology of MAS is complex and still incompletely clarified. Several mechanisms are implicated in the pathogenesis of hypoxemia and decreased lung compliance caused by meconium aspiration.

- Airway obstruction: Depending on consistency and amount, particulate meconium may mechanically obstruct the airways, completely resulting in atelectasis and ventilation perfusion mismatch or partially causing ball valve air trapping and air leak syndromes.

- Surfactant inactivation: Meconium components (cholesterol and bile acids) inactivate lung surfactant by changing the viscosity and ultrastructure of surfactant, fragmentation of dipalmitoylphosphatidylcholine and accelerating its conversion from large, surface-active aggregates into small, less active forms. It also decreases the levels of surfactant proteins A and B and directly harms type II pneumocytes, thereby decreasing surfactant production. ${ }^{20}$

- Chemical irritation: Meconium is a chemoattractant for neutrophils and macrophages resulting in their accumulation in alveoli and airways within hours of aspiration. The ensuing release of proinflammatory cytokines, such as tumor necrosis factor- $\alpha$, interleukin- $1 \beta$, and interleukin-8, may directly injure lung parenchyma or lead to vascular leakage, causing a toxic pneumonitis with hemorrhagic pulmonary edema. ${ }^{3}$ Apoptosis, cellular infiltration, activation of alveolar macrophages with generation of superoxide anions, increased airway responsiveness or increased cytokine concentrations (IL6 and pancreatic phospholipase A2) in bronchoalveolar lavage of animal lungs instilled with meconium have been demonstrated in numerous studies. ${ }^{21-23} \mathrm{~A}$ recent study by Hofer et al demonstrated that severe MAS is closely linked with high values of C-reactive protein and low total leucocyte and absolute neutrophils count, indicating the role of inflammation in the pathogenesis of MAS. ${ }^{24}$

- Complement activation: A recent hypothesis suggests that meconium activates the two main recognition systems of innate immunity, toll-like receptors and complement system, leading not only to lung dysfunction but also to a systemic inflammatory response. ${ }^{23,25}$ Despite an increasing focus on lung inflammation in MAS, the cellular mechanisms initiating this inflammatory cascade remain to be clarified.
- Persistent pulmonary hypertension (PPHN): Hypoxiainduced pulmonary arterial vasoconstriction contributes to the development of PPHN which is a leading cause of mortality in severe MAS. In addition, vasoactive inflammatory substances-mediated pulmonary vasoconstriction and chronic hypoxia-mediated abnormal muscularization and architectural changes of pulmonary vessels contribute to perinatal maladaptation of pulmonary circulation, resulting in pulmonary hypertension. ${ }^{26}$

- Infection: Prior experimental work has shown that meconium enhances bacterial growth in vitro and the risk of intraamniotic infection (both clinical and culture proven) is increased in the presence of MSAF. ${ }^{27}$ However, a clear relationship between MAS and sepsis has not been documented till date.

Meconium also has a detrimental effect on placental and umbilical tissues, resulting in vasoconstriction, ulceration, and vascular necrosis, potentially compromising fetal oxygenation. ${ }^{26}$ It inhibits fetal lung fluid reabsorption at birth through unknown mechanisms, disturbing the ability of the lungs to adapt properly to extrauterine life. ${ }^{28}$ Further detailed studies to elucidate the changing concepts in pathophysiology of MAS from mechanical obstruction to the role of immunoinflammatory mechanisms are required for exploring newer strategies in the management of MAS.

\section{Clinical features}

Neonates with MAS often display the classic signs of postmaturity with peeling of the skin, long fingernails, weight loss, and decreased vernix. The vernix, nails, and skin become meconium-stained depending upon the time of exposure; nails become stained after 6 hours and vernix after 12-14 hours of exposure. ${ }^{2}$ Approximately $20 \%-33 \%$ of them exhibit neurologic and/or respiratory depression at birth typically due to hypoxia or shock. ${ }^{29}$

Respiratory distress with marked tachypnea, retractions, grunting, nasal flaring, and cyanosis ensues soon after birth. Affected infants typically have a barrel-shaped chest with an increased anterior-posterior diameter caused by overinflation. Chest auscultation reveals rales and rhonchi. Patients with severe disease are at risk for respiratory failure, which is often associated with PPHN, right-to-left shunting, and air leaks (pneumothorax or pneumomediastinum). A few patients, asymptomatic at birth, develop worsening signs of respiratory distress later as the meconium moves from the large airways into the lower tracheobronchial tree. Hence, any infant born through MSAF should be monitored for at least 24 hours. 


\section{Diagnosis}

Despite extensive research, the diagnostic criterion for MAS is not well defined. MAS is said to occur in any neonate born through MSAF with respiratory distress at or shortly after birth. The initial chest film may show streaky linear densities (similar to transient tachypnea of the newborn) progressing to hyperinflation with diffuse patchy densities. ${ }^{30}$ Air leak occurs in $10 \%-30 \%$ of infants with MAS. Arterial blood gas measurements typically demonstrate hypoxemia and hypercarbia. However, these findings are nonspecific and are not used to diagnose MAS but to assess the respiratory status of the infant.

The differential diagnosis of MAS includes other causes of respiratory distress which occur in 4\%-9\% cases of MSAF. These are transient tachypnea of newborn (occurs in 34-37 weeks of gestation and usually resolves within 24 hours), delayed transition from fetal circulation, sepsis or pneumonia, persistent pulmonary hypertension, and congenital cyanotic heart disease.

\section{Management}

The management of MAS is primarily supportive with no definite proven therapy. Broadly, it can be divided into preventive strategies and neonatal management.

\section{Prevention of MAS}

MAS is frequently associated with a vicious cycle of hypoxemia, shunting, acidosis, and pulmonary hypertension. Much of the morbidity associated with MAS is secondary to related problems such as asphyxia, sepsis, and PPHN. Therefore, the aim of intervention in the delivery room should be directed to reduce the incidence and severity of meconium aspiration and these comorbidities. Over the last decade, interventions such as elective reduction of post-term births, aggressive management of fetal distress, and reduced incidence of birth asphyxia have resulted in a significant decline in MAS incidence. ${ }^{31}$

Elective induction of labor at 41 weeks of gestation prevents maturation-induced meconium passage and possible asphyxia because of failing placental function resulting in secondary MAS. A recent Cochrane review of 19 trials showed that elective induction at 41 weeks of gestation reduced the risk of perinatal mortality with lower incidence of MAS in the induced group (relative risk: $0.29,95 \%$ CI: $0.12-0.68$ ). ${ }^{32}$ Similar results have been suggested by the meta-analysis of 14 randomized controlled trials (RCTs) highlighting the benefits of this intervention. ${ }^{33}$ Continuous or periodic intrapartum fetal heart rate monitoring has been advocated as standard of care in high-risk pregnancies. Fetal distress precedes MAS and this may be indicated by a low umbilical $\mathrm{pH}$ and/or APGAR score at $5 \mathrm{~min}$. Fetal pulse oximetry, approved by US Food and Drug Administration (FDA), can be done only after the rupture of membranes with a cervical dilation of $2-3 \mathrm{~cm}$. In cases of nonreassuring fetal heart rate tracings, a high correlation between fetal oxygen saturation below $30 \%$ and scalp pH value of 7.2 have been shown, though with the current technology, adequate signals can be obtained only $70 \%$ of the times. ${ }^{34}$

Intrapartum amnioinfusion using isotonic sterile solution (normal saline or ringer lactate) has been proposed to reduce the incidence and severity of MAS by the dilution of thick meconium, thereby decreasing its consistency, and relief of cord compression, oligohydramnios, and thus, fetal distress. A meta-analysis of 13 studies demonstrated that prophylactic intrapartum amnioinfusion for moderate or thick MSAF significantly reduced the frequency of MAS, cesarean section rate, meconium below the vocal cords, and neonatal acidemia with no increase in chorioamnionitis. ${ }^{35}$ However, the benefits of this intervention have been found to be conflicting with the risk of potential complications such as chorioamnionitis, premature rupture of membranes, placental abruption, preterm labor, cord hemorrhage, cord prolapse, amniotic fluid embolism, and maternal death. ${ }^{36}$ The American College of Gynecology and Obstetrics has stated that the routine use of amnioinfusion for dilution of MSAF is not recommended. ${ }^{37}$ A Cochrane meta-analysis review suggests amnioinfusion to be considered for women with meconium-stained liquor in units with limited facilities for peripartum surveillance, and with no demonstrable reduction in MAS incidence in settings with standard peripartum surveillance..$^{38}$

Currently, meconium staining can be diagnosed only by direct visualization of the amniotic fluid at the time of rupture of membranes. A diagnostic process that would allow a noninvasive means of detecting meconium staining in a term pregnancy is being evaluated. This would involve noninvasive illumination of the amnion with light of a specific wavelength and detection of the fluorescence of certain biological pigments contained within the meconium. ${ }^{39}$

\section{Delivery room management}

The recommendations for delivery room management of meconium-stained newborns have undergone significant changes during the last four decades on the basis of several observational studies, RCTs, and systematic reviews.

\section{Intrapartum oropharyngeal suctioning}

This was considered a standard procedure for more than 25 years on the basis of the seminal work of Carson et al, 
who concluded that routine intrapartum pharyngeal suctioning of meconium-stained neonate significantly reduced the incidence and severity of MAS. ${ }^{40} \mathrm{~A}$ series of large, prospective RCTs in the last decade brought landmark changes to this approach by demonstrating no benefit of such an intervention. Vain et al, comparing intrapartum suction with no suction in meconium-stained neonates, showed no difference in the incidence of MAS, mortality, need for mechanical ventilation, and the duration of oxygen therapy. ${ }^{41}$ Another study by Nangia et al conducted in resource-poor settings with limited facilities for ante/ intrapartum fetal surveillance also had similar results. ${ }^{42}$ Following these, routine intrapartum oropharyngeal suctioning in vigorous meconium-stained neonates was removed from the NRP 2005 guidelines and is no longer practiced. $^{43}$

\section{Postpartum endotracheal suctioning}

Intubation and tracheal toileting have remained a matter of debate till the most recent times. Traditionally done in all neonates with meconium staining, the practice did not confer any additional benefits over expectant management in preventing MAS in vigorous nondepressed babies in a large multicenter RCT. ${ }^{29} \mathrm{~A}$ Cochrane meta-analysis of four RCTs also did not show a difference in MAS incidence between intubated and nonintubated vigorous meconium-stained neonates. ${ }^{44}$ Subsequently, as per NRP 2010 , the practice was restricted to nonvigorous meconium-stained babies due to lack of evidence on any harm or benefits of this procedure in the aforementioned group. ${ }^{45}$

Recent pilot trials by Nangia et al and Chettri et al have concluded that endotracheal suction does not appear to alter the incidence of MAS/death even in nonvigorous meconium-stained infants. ${ }^{46,47}$ Further, no difference was found between two groups in other parameters like severity and duration of respiratory distress, need for respiratory support, incidence of hypoxic ischemic encephalopathy, duration of oxygen therapy, and duration of hospitalization. The most recent ILCOR guidelines 2015 thereby recommend that nonvigorous newborns with MSAF do not require routine intubation and tracheal suctioning. ${ }^{48}$

An institutional policy that provides for a well-coordinated and trained "rapid response team" consisting of obstetricians, perinatal nurses, and pediatricians for meconium-stained births is likely to implement an evidence-based approach and effectively manage such events, thereby reducing the incidence of MAS and requirement for costly and sophisticated interventions. ${ }^{5}$

\section{Neonatal management}

All neonates at risk of MAS who show respiratory distress should be admitted to a neonatal intensive care unit and monitored closely. The treatment is mainly supportive and aims to correct hypoxemia and acidosis with the maintenance of optimal temperature and blood pressure.

\section{General management}

All infants should be monitored using noninvasive monitors and blood gas sampling through an indwelling central arterial catheter. Minimal handling, maintaining a noise-free environment, sedation, and analgesia (using opioids) are used frequently to prevent pain-and stress-induced hypoxia and right-to-left shunting in ventilated patients with MAS Other effective steps in management include maintenance of normothermia, correction of metabolic abnormalities (hypoglycemia, hypocalcemia, acidosis, and polycythemia), and maintenance of systemic blood pressure greater than pulmonary blood pressure by volume expansion, transfusion or vasopressors.

\section{Nasogastric aspiration}

Prophylactic nasogastric lavage before first feed has been advocated to prevent feed intolerance and secondary MAS due to postnatal aspiration of gastric contents. However, in a large RCT study done at our center in vigorous babies with MSAF, no difference in the incidence of feeding problems or secondary MAS was observed between the lavage and nonlavage group. ${ }^{49}$ Similar results have been shown in other trials with no clear demonstrable benefits of this practice. ${ }^{50,5}$ Most of these studies have included only vigorous neonates who might not have had prolonged meconium exposure in utero. In a recent meta-analysis, Deshmukh et al, inferred that prophylactic lavage reduces the risk of feed intolerance in meconium-stained infants by $30 \%{ }^{52}$ Hence, evidence of use of this procedure in nonvigorous neonates needs to be addressed by means of further studies.

\section{Respiratory support}

The requirement of respiratory support varies according to the severity of MAS, with a majority of neonates responding to only supplemental oxygen administration by hood or nasal prongs. The target oxygen saturation is maintained between $90 \%$ and $95 \%$, given the high incidence of rightto-left shunting. About $10 \%$ of those with severe MAS are treated by CPAP alone, delivered by binasal or single nasal prongs at a pressure of $5-8 \mathrm{~cm} \mathrm{H}_{2} \mathrm{O} .{ }^{8}$ However, tolerance to CPAP device is often limited due to the relative maturity 
of infants and chances of resulting agitation worsening pulmonary hypertension, thereby becoming an indication of intubation. ${ }^{29}$

Approximately $40 \%$ of neonates with MAS require intubation and mechanical ventilation. The indications for intubation include high oxygen requirement (fraction of inspired oxygen $\left[\mathrm{FiO}_{2}\right]>0.8$ ), respiratory acidosis (arterial $\mathrm{pH}$ persistently $<7.25$ ), pulmonary hypertension, and circulatory compromise. ${ }^{53}$ Despite more than four decades of ventilation of MAS infants, the optimal ventilatory management remains controversial with very few clinical trials upon which to base definite recommendations. This ensues because of complex pathophysiology involving areas of atelectasis coexisting with hyperinflation along with ventilation perfusion mismatch and airway compromise. The goal for assisted ventilation is to achieve optimal gas exchange with minimal lung barotrauma. Though trials regarding the appropriate mode of ventilation are lacking, synchronized intermittent mandatory ventilation is the most commonly used mode in conventional ventilation. Ventilator settings and target blood gas depend upon the presence or absence of PPHN and predominant lung abnormality. In cases with marked regional or global atelectasis, high-peak inspiratory pressure (PIP) (up to maximum $30 \mathrm{~cm}$ ) and high positive-end expiratory pressure (PEEP) $(4-7 \mathrm{~cm})$ with longer inspiratory time helps in the recruitment of alveoli. If there is obvious gas trapping, PEEP should be decreased $(3-4 \mathrm{~cm})$ with optimal expiratory time $(0.5-0.7)$ and rapid ventilator rates. ${ }^{54}$ In infants with associated PPHN, higher rates $(50-70)$ with higher $\mathrm{FiO}_{2}(80 \%-100 \%)$ should be considered to maintain $\mathrm{PaO}_{2}$ between 70 and $100 \mathrm{~mm} \mathrm{Hg}$ and $\mathrm{PaCO}_{2}$ between 35 and $45 \mathrm{~mm} \mathrm{Hg}$ along with volume expansion, vasopressors, and other supportive measures..$^{53,54}$ Hyperventilation-induced alkalosis should be avoided due to risk of cerebral vasoconstriction-induced neurologic injury and sensorineural hearing loss. In such situations, other modalities like inhaled nitric oxide and high-frequency ventilation should be considered early.

\section{High-frequency ventilation}

Despite a dearth of trial-based evidence, high-frequency ventilation (HFV) has been found beneficial in treating patients with severe MAS failing conventional ventilation. It provides effective gas exchange at low tidal volume, thus decreasing barotrauma and air leaks. Published series from large neonatal databases suggest that $20 \%-30 \%$ of all infants requiring intubation and ventilation with MAS are treated with high-frequency ventilation. ${ }^{55}$ High frequency oscillatory ventilation is the more commonly used mode, especially in those with significant atelectasis, where application of higher mean airway pressures (around $25 \mathrm{~cm}$ ) with recruitment maneuvers at moderate frequency $(6-8 \mathrm{~Hz})$ has been found to be beneficial. ${ }^{54}$ It also lends a clinical advantage in MAS with associated severe PPHN, as the response to inhaled NO is better with HFOV than conventional ventilation, with recent evidence suggesting failure of this combination and requirement of ECMO in only $5 \%$ of such babies. ${ }^{54}$ High frequency jet ventilation has also been found to be beneficial in optimizing oxygenation and prevention of ECMO in severe MAS especially with an underlying combination of atelectasis and gas trapping. ${ }^{56}$ Hence, high-frequency ventilation should be considered over conventional pneumothorax in settings of severe MAS with intractable hypoxemia, persistent respiratory acidosis, or high risk of pneumothorax.

\section{Surfactant therapy}

The detrimental effect of meconium on surfactant production and function has been well known and forms the basis of the use of exogenous surfactant in severe MAS. Canadian Pediatric Society recommends exogenous surfactant for all intubated patients with MAS with $\mathrm{FiO}_{2}$ requirement $>50 \%$. Surfactant can be given either as a bolus or in the form of bronchoalveolar lavage. In the developed world, bolus surfactant therapy is currently used in $30 \%-50 \%$ of ventilated infants with MAS. ${ }^{8}$ A meta-analysis of four RCTs involving 326 MAS infants showed reduction in the severity of respiratory illness and in the requirement of ECMO with surfactant administration, but no significant effect on overall mortality. ${ }^{57}$ Clinical trials of surfactant lung lavage have suggested that lavage therapy may improve oxygenation and shorten the duration of ventilation. ${ }^{58}$ However, a meta-analysis of two RCTS did not show any significant improvement in mortality, pneumothorax, duration of ventilation, or duration of hospitalization. ${ }^{59}$ In another RCT, authors demonstrated no effect of lavage on pulmonary outcomes but found a higher rate of ECMO free survival in the treated group. ${ }^{60} \mathrm{~A}$ recent systematic review evaluating the effect of surfactant administration either as bolus or lavage concluded that both decreased the duration of hospital stay, the duration of mechanical ventilation, and the need for ECMO with no reduction in mortality. ${ }^{61}$ Hence, surfactant therapy should be used judiciously in MAS choosing infants with severe disease and treating early and if necessary, repeatedly, for optimum results. Further trials are needed to confirm the treatment effect, define the optimal doses and method of surfactant administration, and compare lavage with bolus administration. 


\section{Corticosteroids}

Pulmonary and systemic inflammation is a key feature of MAS pathophysiology providing the rationale behind the use of steroids in cases of severe MAS to improve pulmonary function. According to Cochrane meta-analysis, use of steroids was not associated with a decrease in mortality, the duration of mechanical ventilation, or the duration of hospitalization but showed an increase in the duration of oxygen therapy. ${ }^{62}$ Recently, trials using corticosteroids have shown shortened duration of stay and oxygen dependence with improved radiological clearance in the steroid-treated group. ${ }^{63,64}$ In the absence of conclusive evidence, routine steroid therapy is not recommended in MAS. In a few animal studies, inhaled budesonide has shown a promising response when instilled intratracheally alone or with surfactant, thus providing a promising future therapy. ${ }^{65,66}$ More evidencebased studies to evaluate the potential benefits and harms of steroid therapy in MAS are the need of the hour.

\section{Antibiotics}

The association between meconium and sepsis has been evaluated extensively, but the role of antibiotics in the routine management of MAS still remains controversial. In a RCT study on term babies born through MSAF conducted by the author, routine antibiotic prophylaxis did not reduce the incidence of culture-proven sepsis or MAS in the intervention group. ${ }^{67} \mathrm{~A}$ meta-analysis of three RCTs concluded that the use of antibiotics for MAS did not result in significant reduction in the risk of mortality, sepsis, or duration of hospital stay. ${ }^{61}$ Given the limited number of studies and small number of neonates enrolled, the role of antibiotics needs to be re-evaluated in well-designed trials.

\section{Inhaled nitric oxide (iNO)}

Large RCTs have shown the efficacy of iNO in term infants with PPHN in decreasing mortality and the need for ECMO. ${ }^{68}$ Each of these trials included a large subgroup with MAS. Currently, around $20 \%-30 \%$ of all ventilated infants with MAS receive iNO and around 40\%-60\% show a sustained response. ${ }^{69}$ Being a selective pulmonary vasodilator, it is an ideal treatment for moderate to severe PPHN persisting despite ventilatory maneuvers. In severe PPHN, a synergistic improvement in oxygenation occurs with the use of HFV with iNO owing to decreased pulmonary shunting and increased delivery of iNO to its site of action..$^{70}$ A significant proportion of neonates with PPHN do not respond to iNO. Moreover, the high cost of therapy preludes its routine use in developing countries. Other pulmonary vasodilators like phosphodiesterase 5 inhibitors (sildenafil, dipyridamole, and zaprinast) have been tried in newborns, but the number treated is few, with no large control studies available to date. ${ }^{71}$

\section{Extracorporeal membrane oxygenation (ECMO)}

MAS has been the leading diagnosis of neonates being referred for this therapy, with the usual indication being intractable hypoxemia (persistent oxygenation index $>40$ ) despite optimal ventilation. ${ }^{72}$ With the advent of newer therapies, the number of infants requiring ECMO has reduced drastically, but survival in MAS cases remains high (up to 95\%). ${ }^{72}$ Follow-up studies of ECMO-treated cases demonstrate lower rates of severe disability at 1 year, indicating the beneficial role as the rescue therapy of severe MAS. ${ }^{73}$

\section{Future therapy}

The management of MAS remains essentially supportive with no definite therapy for meconium-induced lung injury. A better understanding of the pathological mechanisms resulting in meconium-induced parenchymal injury has widened the scope of potential future therapies for MAS. Recent data suggest that at least some cases of cell death by meconium occur by apoptosis which is mediated by the renin angiotensin system. Pretreatment of rabbit pup with angiotensin converting enzyme inhibitor captopril before intratracheal instillation of meconium reduced lung epithelial cell apoptosis. ${ }^{74}$ Similarly, the administration of $\mathrm{N}$ acetyl cysteine, along with surfactant, has been shown to prevent neutrophil migration, lung edema, and oxidative damage by suppressing IL- 8 and IL- $\beta$ formation. ${ }^{75}$ The administration of cyclooxygenase II inhibitor parecoxib in meconium-treated rabbits reduces the expression of myeloperoxidase and COX II receptors and attenuates histopathologic damage and meconium-induced acute lung injury with significant improvement in respiratory functions ${ }^{76}$ Rescue therapy with intratracheal albumin also improves lung function by binding and blocking active substances like free fatty acids and bile acids in meconium. ${ }^{77}$ A protease inhibitor cocktail prevents cell detachment induced by meconium, suggesting a possible role of fetal pancreatic digestive enzymes in the treatment of MAS. ${ }^{78}$ Liquid ventilation with perfluorocarbons has been found beneficial in improving survival in animal models of MAS, offering research potential for its use in humans. ${ }^{79} \mathrm{All}$ these therapies are in experimental stage, with only animal studies available so far, and await further human trials. 


\section{Outcome}

Refinements in neonatal intensive care facilities and newer therapies including respiratory support have resulted in marked improvement in the overall outcome of MAS. A retrospective review of term babies with MAS between 1997 and 2007 showed a mortality rate of $1.2 \%$, as compared to $4.2 \%$ reported previously (1973 to 1987 ). In the same study, APGAR score $<3$, the need for ventilator support within 48 hours, the repeated dose of vasopressor agents, and the use of cefotaxime were found to be independent risk factors for mortality. ${ }^{80}$ In a recent trial from India, mortality rate was found to be as high as $26 \%$, with lower birth weight, the presence of myocardial dysfunction, and higher initial oxygen requirement being independent predictors of mortality. ${ }^{81}$ Approximately, one third of these deaths in MAS are directly attributable to pulmonary cause, with the remainder being ascribed to accompanying hypoxic ischemic encephalopathy.

The short-term morbidities include pneumothorax and other air leaks in up to $10 \%$ of ventilated patients. ${ }^{8}$ Others like oxygen supplementation at 28 days (5\%), seizures (4\%), or pulmonary hemorrhage occur in only in a small proportion of infants. ${ }^{8,80}$ Long-term pulmonary morbidity, especially in the form of reactive airway disease, is common, with about half of babies developing episodes of coughing/wheezing during infancy. ${ }^{82}$ Older children are likely to have mild airway obstruction, hyperinflation, and airway hyper-responsiveness with normal pulmonary function test and chest X-ray. ${ }^{83}$ In contrast, the neurological outcome is more dismal, with about $21 \%$ of infants developing cerebral palsy or global developmental delay despite responding to conventional ventilation alone. ${ }^{84}$ A poor neurological outcome (10\%-20\% developmental disability) irrespective of the mode of delivery, milder course of illness, or the mode of ventilation demonstrates that predisposing factors and underlying factors leading to MAS are fundamental to the pathogenesis of neurodevelopment handicap among the survivors. ${ }^{84}$ With improvement in mortality, more robust follow-up studies are required to study these factors and long-term neurodevelopmental outcome in these patients.

\section{Conclusion}

Advances in obstetrical and neonatal management practices have led to marked improvement in morbidity and mortality associated with MAS. However, lack of novel therapeutic facilities and protocolized management guidelines is still a major problem in dealing with this common condition in developing countries. Supportive care is the cornerstone of MAS management, and with its judicial use, infants with even severe MAS survive with an acceptable burden of short-and long-term morbidity. Further studies should be undertaken to provide a deeper insight into its complex pathophysiology and evaluation of newer therapies, with potential benefits documented in preliminary animal trials.

\section{Disclosure}

The authors report no conflicts of interest in this work.

\section{References}

1. Antonowiez I, Schwachman H. Meconium in health and disease. Adv Paediatr. 1979;26:275-310.

2. Fanaroff AA. Meconium aspiration syndrome: historical aspects. $J$ Perinatol. 2008;28 (Suppl 3):S3-S7.

3. Cleary GM, Wiswell TE. Meconium-stained amniotic fluid and the meconium aspiration syndrome: an update. Pediatr Clin North Am. 1998;45(3):511-529.

4. Fischer C, Rybakowski C, Ferdynus C, Sagot P, Gouyon JB. A population based study of meconium aspiration syndrome in neonates born between 37 and 43 weeks gestation. Int J Pediatr. 2012;321545.

5. Bhutani VK. Developing a systems approach to prevent meconium aspiration syndrome: lessons learned from multinational studies. $J$ Perinatol. 2008;28 (Suppl 3):S30-S35.

6. Poggi SH, Ghidini A. Pathophysiology of meconium passage into the amniotic fluid. Early Hum Dev. 2009;85(10):607-610.

7. Sankhyan N, Sharma VM. Predictors of meconium stained amniotic fluid: a possible strategy to reduce neonatal morbidity and mortality. $J$ Obstet Gynecol India. 2006;56(6):514-517.

8. Dargaville PA, Copnell B; Australian and New Zealand Neonatal Network. The epidemiology of meconium syndrome: incidence, risk factors, therapies and outcome. Pediatrics. 2006;117(5):1712-1721.

9. Wiswell TE. Delivery room management of the meconium-stained newborn. J Perinatol. 2008;28 (Suppl 3):S19-S26.

10. Gelfand SL, Fanaroff JM, Walsh MC. Meconium stained fluid: approach to the mother and baby. Pediatr Clin North Am. 2004;51(3):655-667.

11. Liu WF, Harrington T. Delivery room risk factors for meconium aspiration syndrome. Am J of Perinatol. 2002;19(7):367-378.

12. Usta IM, Mercer BM, Sibai BM. Risk factors for meconium aspiration syndrome. Obstetgynecol. 1995;86(2):230-234.

13. Yoder BA, Kirsch EA, Barth WH, Gordon MC. Changing obstetric practices associated with decreasing incidence of meconium aspiration syndrome. Obstet Gynecol. 2002;99(5):731-739.

14. Ballard RA, Hansen TN, Corbet A. Respiratory failure in the term infant. In: Taeusch HW, Ballard RA, Gleason CA, editors. Avery's Diseases of the Newborn. 8th ed. Philadelphia: Elsevier Inc; 2005:705-722.

15. Manning FA, Harman CR, Morrison I, Menticoglou SM, Lange IR, Johnson JM. Fetal assessment based on fetal biophysical profile scoring. Am J Obstet Gynecol. 1990;162(3):703-709.

16. Richey SD, Ramin SM, Bawson RE et al. Markers of acute and chronic asphyxia in infants with meconium-stained amniotic fluid. Am J Obstet Gynecol. 1995;172:1212-1215.

17. Ciftci AO, Tanyel FC, Karnak I, Büyükpamukçu N, Hiçsönmez A. In utero defecation: fact or fiction? Eur J Pediatr Surg. 1999;9(6):376-380.

18. Blackwell SC, Moldenhauer J, Hassan SS, et al. Meconium aspiration syndrome in term neonates with normal acid-base status at delivery: is it different? Am J Obstet Gynecol. 2001;184(7):1422-1425.

19. Ghidini A Spong CY. Severe meconium aspiration syndrome is not caused by aspiration of meconium. Am J Obstet Gynecol. 2001;185(4):931-938.

20. Kopincova J, Calkovska A. Meconium-induced inflammation and surfactant inactivation: specifics of molecular mechanisms. Pediatr Res. 2016;79(4):514-521.

21. Zagariya A, Bhat R, Navale S, Vidyasagar D. Cytokine expression in meconium-induced lungs. Indian J Pediatr. 2004;71(3):195-201. 
22. Kojima T, Hattori K, Fujiwara T, Sasai-Takedatsu, M, Kobayashi Y. Meconium induced lung injury mediated by activation of alveolar macrophages. Life Sci. 1994;54(21):1559-1562.

23. Lindenskov PH, Castellheim A, Saugstad OD, Mollnes TE. Meconium aspiration syndrome: possible pathophysiological mechanisms and future potential therapies. Neonatology. 2015;107(3):225-230.

24. Hofer N, Jank K, Strenger V, Pansy J, Resch B. Inflammatory indices in meconium aspiration syndrome. Pediatr Pulmon. 2016;51(6): 601-606

25. Molnes TE, Castellheim A, Lindenskov PH, Salvesen B, Saugstad O. Role of complement in meconium aspiration syndrome. J Perinatol. 2008;28 (Suppl 3):S116-S119.

26. Ahanya SN, Sureshbabu N, Morgan BL, Ross MG. Meconium passage in utero: mechanisms, consequences and management. Obstet Gynecol Surv. 2005;60(1):45-56.

27. Hutton EK, Thorpe J. Consequences of meconium stained amniotic fluid: what does the evidence tell us? Early Hum Dev. 2014;90(7): 333-339.

28. Chua BA, Chan L, Kindler PM, Perks AM. The association between meconium and the production and reabsorption of lung liquid and lactate loss by in vitro lungs from fetal guinea pigs. Am J Obstet Gynecol. 2000;183(1):235-244.

29. Wiswell TE, Gannon CM, Jacob J, et al. Delivery room management of the apparently vigorous meconium-stained neonate: results of the multicenter, international collaborative trial. Pediatrics. 2000;105(1 Pt 1): $1-7$.

30. Gooding CA, Gregory GA. Roentgenographic analysis of meconium aspiration of the newborn. Radiology. 1971;100(1):131.

31. Vain NE, Szyld EG, Prudent LM, Aguilar AM. What (not) to do at and after delivery? Prevention and management of meconium aspiration syndrome. Early Hum Dev. 2009;85(10):621-626.

32. Gulmezoglu AM, Crowther CA, Middleton P. Induction of labor for improving birth outcomes for women at or beyond term. Cochrane Database Syst Rev. 2006;(4):CD004945.

33. Hussain AA, Yakoob MY, Imdad A, Bhutta ZA. Elective induction for pregnancies at or beyond 41 weeks of gestation and its impact on stillbirths: a systematic review with meta-analysis. BMC Public Health. 2011;11 (Suppl 3):S5.

34. Kuhnert M, Seelbach-Goebel B, Butterwegge M. Predictive agreement between the fetal arterial oxygen saturation and fetal scalp pH: results of the German multicenter study. Am J Obstet Gynecol. 1998;178(2):330-335.

35. Pierce J, Gaudier F, Sanchez-Ramos L. Intrapartum amnioinfusion for meconium stained fluid: meta-analysis of prospective clinical trials. Obstet Gynecol. 2000;95(6 Pt 2):1051-1056.

36. Velphi S, Vidyasagar D. Intrapartum and post delivery management of infants born to mother with meconium stained amniotic fluid: evidence based recommendations. Clin Perinatol. 2006;33(1):29-42.

37. ACOG Committee Obstetric Practice. ACOG committee opinion number 346, October 2006: amnioinfusion does not prevent meconium aspiration syndrome. Obstet Gynecol. 2006;108(4):1053.

38. Hofmeyr GJ, Xu H, Eke AC. Amnioinfusion for meconium stained liquor in labour. Cochrane Database Syst Rev. 2014;(1):CD000014.

39. Doody MC, inventor; Doody Michael C, assignee. Prenatal detection of meconium stained amniotic fluid. United States patent US5172693. $1992 \mathrm{Dec} 22$.

40. Carson B, Losey R, Bowes Jr WA, Simmons MA. Combined obstetric and pediatric approach to prevent meconium aspiration syndrome. $\mathrm{Am}$ J Obstet Gynecol. 1976;126(6):712-715.

41. Vain NE, Szyld EG, Prudent LM, Wiswell TE, Aguilar AM, Vivas NI. Oropharyngeal and nasopharyngeal suctioning of meconium stained neonates before delivery of their shoulders: multicentre randomised controlled trial. Lancet. 2004;364(9434):597-602.

42. Nangia S, Pal MM, Saili A, Gupta U. Effect of intrapartumoropharyngeal (IP-OP) suction on meconium aspiration syndrome in developing country: a RCT. Resuscitation. 2015;97:83-87.
43. International Liaison Committee on Resuscitation. The International Liaison Committee on Resuscitation (ILCOR) consensus on science with treatment recommendations for pediatric and neonatal patients: pediatric basic and advanced life support. Pediatrics. 2006;117(5):e955-e977.

44. Halliday HL. Endotracheal intubation at birth for preventing morbidity and mortality in vigorous meconium-stained infants born at term. Cochrane Database Syst Rev. 2001;(1):CD000500.

45. Perlman JM, Wyllie J, Kattwinkel J, et al; Neonatal Resuscitation Chapter Collaborators. Part 11: Neonatal resuscitation: 2010 International consensus on cardiopulmonary resuscitation and emergency cardiovascular care science with treatment recommendations. Circulation. 2010;122(16 Suppl 2):S516-538.

46. Nangia S, Sunder S, Biswas R, Saili A. Endotracheal suction in term non vigorous meconium stained neonates- a pilot study. Resuscitation. 2016;105:79-84

47. Chettri S, Adhisivam B, Bhat BV. Endotracheal suction for nonvigorous neonates born through meconium stained amniotic fluid: a randomized controlled trial. J Pediatr. 2015;166(5):1208-1213.

48. Wyckoff MH, Aziz K, Escobedo MB, et al. Part 13: Neonatal Resuscitation: 2015 American Heart Association Guidelines Update for Cardiopulmonary Resuscitation and Emergency Cardiovascular Care. Circulation. 2015;132(18 Suppl 2):S543-S560.

49. Sharma P, Nangia S, Tiwari S, Goel A, Singla B, Saili A. Gastric lavage for prevention of feeding problems in neonates with meconium-stained amniotic fluid: a randomised controlled trial. PaediatrInt Child Health. 2014;34(2):115-119.

50. Garg J, Masand R, Tomar BS. Utility of gastric lavage in vigorous neonates delivered with meconium stained liquor: a randomized controlled trial. Int J Pediatr. 2014;2014:204807.

51. Ameta G, Upadhyay A, Gothwal S, Singh K, Dubey K, Gupta A. Role of gastric lavage in vigorous neonates born with meconium stained amniotic fluid. Indian J Pediatr. 2013;80(3):195-198.

52. Deshmukh M, Balasubramanian H, Rao S, Patole S. Effect of gastric lavage on feeding in neonates born through meconium-stained liquor: a systematic review. Arch Dis Child Fetal Neonatal Ed. 2015;100(5):F394-F399.

53. Goldsmith JP. Continuous positive airway pressure and conventional mechanical ventilation in the treatment of meconium aspiration syndrome. J Perinatol. 2008;28 (Suppl 3):S49-S55.

54. Dargaville PA. Respiratory support in meconium aspiration syndrome: A practical guide. Int J Pediatr. 2012;2012:965159.

55. Tingay DG, Mills JF, Morley CJ, Pellicano A, Dargaville PA; Australian and New Zealand Neonatal Network. Trends in use and outcome of newborn infants treated with high frequency ventilation in Australia and New Zealand, 1996-2003. J Paediatr Child Health. 2007;43(3):160-166.

56. Engle WA, Yoder MC, Andreoli SP, Darragh RK, Langefeld CD, Hui SL. Controlled prospective randomized comparison of high-frequency jet ventilation and conventional ventilation in neonates with respiratory failure and persistent pulmonary hypertension. J Perinatol. 1997;17(1):3-9.

57. El Shahed AI, Dargaville P, Ohlsson A, Soll RF. Surfactant for meconium aspiration syndrome in full term/near term infants. Cochrane Database Syst Rev. 2007;(3):CD002054

58. Wiswell TE, Knight GR, Finer NN, et al. A multicenter, randomized, controlled trial comparing Surfaxin (Lucinactant) lavage with standard care for treatment of meconium aspiration syndrome. Pediatrics. 2002;109(6):1081-1087.

59. Hahn S, Choi HJ, Soll R, Dargaville PA. Lung lavage for meconium aspiration syndrome in newborn infants. Cochrane Database Syst Rev. 2013;(4):CD003486

60. Dargaville PA, Copnell B, Mills JF, et al; lessMAS Trial Study Group. Randomized controlled trial of lung lavage with dilute surfactant for meconium aspiration syndrome. J Pediatr. 2011;158(3):383-389.

61. Natarajan CK, Sankar MJ, Jain K, Agarwal R, Paul VK. Surfactant and antibiotic therapy in neonates with meconium aspiration syndrome: a systematic review and meta-analysis. J Perinatol. 2016;36 (Suppl 1): S48-S53. 
62. Ward MC, Sinn J. Steroid therapy for meconium aspiration syndrome in newborn infants. Cochrane Database of Systematic Rev. 2003;(4):CD003485.

63. Basu S, Kumar A, Bhatia BD, Satya K, Singh TB. Role of steroids on the clinical course and outcome of meconium aspiration syndrome-a randomized controlled trial. J Trop Pediatr. 2007;53(5):331-337.

64. Tripathi S, Saili A. The effect of steroids on the clinical course and outcome of neonates with meconium aspiration syndrome. J Trop Pediatr. 2007;53(1):8-12.

65. Mokra D, Mokry J, Drgova A, Petraskova M, Bulikova J, Calkovska A. Intratracheally administered corticosteroids improved the lung functions in meconium-instilled rabbits. J Physiol Pharmacol. 2007;58 (Suppl 5 Pt 1):389-398.

66. Mikolka P, Mokra D, Kopinkova J, Tomčíková-Mikušiaková L, Calkovská A. Budesonide added to modified porcine surfactant curosurf may additionally improve the lung functions in meconium aspiration syndrome. Physiol Res. 2013;62 (Suppl 1):S191-S200.

67. Goel A, Nangia S, Saili A, Garg A, Sharma S, Randhawa VS. Role of prophylactic antibiotics in neonates born through meconium stained amniotic fluid (MSAF)-a randomized controlled trial. Eur J Pediatr. 2015;174(2):237-243.

68. Finer NN, Barrington KJ. Nitric oxide for respiratory failure in infants born at or near term. Cochrane Database Syst Rev. 2006;(4):CD000399.

69. Gupta A, Rastogi S, Sahni R, et al. Inhaled nitric oxide and gentle ventilation in the treatment of pulmonary hypertension of the newborn-a single-center, 5-year experience. J Perinatol. 2002;22(6):435-441.

70. Kinsella JP, Truog WE, Walsh WF, et al. Randomized, multicenter trial of inhaled nitric oxide and high-frequency oscillatory ventilation in severe, persistent pulmonary hypertension of the newborn. J Pediatr. 1997; 131(1):55-62.

71. Asad A, Bhat R. Pharmacotherapy for meconium aspiration. J Perinatol. 2008;28 (Suppl 3):S72-S78.

72. Short BL. Extracorporeal membrane oxygenation: use in meconium aspiration syndrome. J Perinatol. 2008:28 (Suppl 3):S79-S83.

73. Mugford M, Elbourne D, FieldD. Extracorporeal membrane oxygenation for severe respiratory failure in newborn infants. Cochrane Database Syst Rev. 2008;(3):CD001340.
74. Uhal BD, Abdul-Hafez A. Angiotensin II in apoptotic lung injury: potential role in meconium aspiration syndrome. J Perinatol. 2008:28 (Suppl3): S108-S112.

75. Kopincova J, Mokra D, Mikolka P, Kolomazník M, Čalkovská A. $\mathrm{N}$-acetylcysteine advancement of surfactant therapy in experimental meconium aspiration syndrome: possible mechanisms. Physiol Res. 2014;63 (Suppl 4):S629-S642.

76. Li A, Zhang L, Li W. Amelioration of meconium-induced acute lung injury by parecoxib in a rabbit model. Int J Clin Exp Med. 2015;8(5):6804-6812.

77. Saugstad OD, Tollofsrud P, Lindenskov P, Drevon CA. Toxic effects of different meconium fractions on lung function: new therapeutic strategies for meconium aspiration syndrome? J Perinatol. 2008;28 (Suppl 3): S113-S115.

78. Ivanov VA, Gewolb IH, Uhal BD. A new look at the pathogenesis of the meconium aspiration syndrome: a role for fetal pancreatic proteolytic enzymes in epithelial cell detachment. Pediatr Res.2010;68(3): 221-224.

79. Jeng MJ, Soong WJ, Lee YS, et al. Effects of therapeutic bronchoalveolar lavage and partial liquid ventilation on meconium-aspirated newborn piglets. Crit Care Med. 2006;34(4):1099-1105.

80. Singh BS, Clark RH, Powers RJ, Spitzer AR. Meconium aspiration syndrome remains a significant problem in the NICU: outcomes and treatment patterns in term neonates admitted for intensive care during a ten-year period. J Perinatol. 2009;29(7):497-503.

81. Louis D, Sundaram V, Mukhopadhay K, Dutta S, Kumar P. Predictors of mortality in neonates with meconium aspiration syndrome. Indian Pediatr. 2014;51(8):637-640.

82. Yuksel B, Greenough A, Gamsu HR. Neonatal meconium aspiration syndrome and respiratory morbidity during infancy. Pediatr Pulmonol. 1993;16(6):358-361.

83. Swaminathan S, Quinn J, Stabile MW, Bader D, Platzker AC, Keens TG. Long-term pulmonary sequelae of meconium aspiration syndrome. J Pediatr. 1989;114(3):356-361.

84. Beligere N, Rao R. Neurodevelopmental outcome of infants with meconium aspiration syndrome: report of a study and literature review. J Perinatol. 2008;28 (Suppl 3):S93-S101.
Research and Reports in Neonatology

\section{Publish your work in this journal}

Research and Reports in Neonatology is an international, peer-reviewed, open access journal publishing original research, reports, editorials, reviews and commentaries on neonatal health. The manuscript management system is completely online and includes a very quick and fair peer-review system. Visit http://www.dovepress.com/testimonials.php to read real quotes from published authors. 